\title{
Effect of Contact Angle on Steam Dropwise Condensation: A Simulation Approach
}

\author{
Milad Nahavandi and Arjomand Mehrabani-Zeinabad \\ Department of Chemical Engineering, Isfahan University of Technology, Isfahan 84156-83111, Iran \\ Correspondence should be addressed to Milad Nahavandi, m.nahavandi@ce.iut.ac.ir
}

Received 22 January 2012; Accepted 14 February 2012

Academic Editors: C.-T. Hsieh and M. E. R. Shanahan

Copyright ( $\odot 2012$ M. Nahavandi and A. Mehrabani-Zeinabad. This is an open access article distributed under the Creative Commons Attribution License, which permits unrestricted use, distribution, and reproduction in any medium, provided the original work is properly cited.

\begin{abstract}
Dropwise condensation process on surface of a vertical polytetrafluouroethylene (PTFE) plate at atmospheric pressure was simulated. Comparison of simulated data with experimental and theoretical results indicates that performed simulation results confirm experimental data, although they deviated from existing proposed correlations. For calculation of heat transfer coefficient and droplets size distribution, simulation of condensation process over vertical copper and PTFE surfaces at atmospheric pressure was performed. By considering the effect of contact angle on heat transfer resistances of droplets, the gained data were optimized in order to evaluate droplets size distribution coefficient. This distribution coefficient was used in a new correlation for prediction of heat transfer coefficient for dropwise condensation process. Comparison of experimental results with the correlation shows a good agreement, $11 \%$ relative error.
\end{abstract}

\section{Introduction}

Condensers are used in various processing industries, and their efficiency improvement has a crucial role not only in optimization of energy consumption, but also on protection of environment. Enhancement of efficiency of this equipment requires full knowledge of condensation process, which is in conjunction with various phenomena such as heat transfer, phase change, and fluid dynamics. Occurrence of different types of condensation process, filmwise and dropwise, depends on existing operational and condensing surface conditions. Heat transfer coefficient of dropwise condensation is much higher than the other type, and this issue makes it very attractive. Condensing surface properties play an important role in type of condensation, heat transfer rate, condenser efficiency, and consequently operating costs. The rate of mass transfer to liquid-vapor interface was evaluated by Schrage based on the Maxwell velocity distribution theory. Based on the developed correlation, the flux of formed droplets on the cooled surface is the difference between surface adsorption of vapor and liquid desorption fluxes $[1,2]$ :

$$
\dot{m}=\frac{2 \sigma}{2-\sigma} \frac{h_{l v}}{\sqrt{2 \pi R}} \frac{\left(T_{v}-T_{i}\right)}{v_{l} T_{v}^{3 / 2}},
$$

where $\sigma$ is the condensation coefficient, $T_{v}$ and $T_{i}$ are the vapor and liquid-vapor interfacial temperatures, respectively, $h_{l v}$ is the condensation latent heat, $v_{l}$ is the specific volume of condensate, and $R$ is the universal gases constant.

The type of steam condensation on cooled surface depends on surface properties such as roughness, surface free energy, vapor-surface temperature gradient, and slope of surface. Researchers developed relations for dropwise condensation heat transfer coefficient based on simple hypotheses. Since droplets with different diameters are formed in dropwise condensation, the overall heat transfer coefficient depends on the formed droplets size distribution. LeFere and Rose considered this issue in presenting a relation for overall heat transfer coefficient [1]. Carey obtained following equation for the dropwise condensation heat transfer coefficient according to vapor-surface thermal resistances $\left(\mathrm{W} / \mathrm{m}^{2} \cdot \mathrm{K}\right)$ $[3,4]$ :

$$
h=\frac{\pi}{2} \int_{D_{\min }}^{D_{\max }} n_{D}^{\prime \prime} D^{2} \frac{\left(1-\left(D_{\min } / D\right)\right)}{\left(1 / h_{i}+D / 4 k_{l}\right)} d D,
$$

where $D_{\min }$ and $D_{\max }$ are the minimum and maximum droplets diameters on the cooled surface, $n_{D}^{\prime \prime}$ is the droplets distribution coefficient, and $k_{l}$ is the thermal conductivity of 
condensate. $h_{i}$ is the apparent heat transfer coefficient at the liquid-vapor interface and is given by $[2,5]$

$$
h_{i}=\left(\frac{2 \sigma}{2-\sigma}\right) \frac{h_{l v}^{2}}{T_{v} v_{l}}\left(\frac{M}{2 \pi R T_{v}}\right)^{1 / 2}
$$

where $M$ is the fluid molecular weight.

Griffith proposed the following correlation to evaluate dropwise condensation heat transfer coefficient $\left(\mathrm{W} / \mathrm{m}^{2} \cdot \mathrm{K}\right)$ $[3,6]$ :

$$
h=\left\{\begin{array}{l}
51104+2044 T^{\text {sat }} \longrightarrow 22^{\circ} \mathrm{C}<T^{\text {sat }}<100^{\circ} \mathrm{C}, \\
255510 \longrightarrow T^{\text {sat }}>100^{\circ} \mathrm{C} .
\end{array}\right.
$$

So far, the effect of contact angle in calculation of dropwise condensation heat transfer coefficient was not considered in any published relationship. This paper discusses the modification of (2) in order to include the effect of this parameter. Furthermore, the droplets' size distribution coefficient as an unknown parameter of (2) was obtained by simulation for dropwise condensation of steam on the PTFE vertical surface at pressure of $1 \mathrm{~atm}$.

\section{Modeling and Simulation}

2.1. Model Definition. Geometry and initial conditions of the selected condensation system are shown in Figure 1. For performing simulation, initially, saturated vapor at 1 atm was contacted with a cold PTFE wall. This leads to occurrence of a phase change and formation of condensate droplets.

2.2. Subdomain Equations. Interface dynamics can be described by phase field equation, derived by Cahn-Hilliard [6]

$$
\left(\frac{\partial \phi}{\partial t}+(U \cdot \nabla) \phi\right)=\nabla \cdot \gamma \nabla G+\dot{\phi}
$$

where $\phi$ is the phase field variable, $G$ is the chemical potential, $\gamma$ is the phase field mobility parameter, and $\dot{\phi}$ is the rate of phase generation.

The velocity field and pressure for the liquid phase were described by the incompressible Navier-Stokes equations:

$$
\begin{aligned}
\rho_{l} \frac{\partial u_{l}}{\partial t}+\rho_{l}\left(u_{l} \cdot \nabla\right) u_{l}= & \nabla \cdot\left[-p_{l} I+\eta_{l}\left(\nabla u_{l}+\left(\nabla u_{l}\right)^{T}\right)\right] \\
& +\rho_{l} g+F_{\text {st }} \\
\nabla \cdot u_{l}= & \dot{m} \delta\left(\frac{1}{\rho_{v}}-\frac{1}{\rho_{l}}\right),
\end{aligned}
$$

where $F_{\text {st }}=G \nabla \phi$ is the surface tension force, $\rho_{l}$ is the liquid density, $u_{l}$ is the liquid velocity, $\eta_{l}$ is the liquid viscosity, and $\delta$ is a smoothed representation of the interface between the two phases.
For the vapor phase, weakly compressible Navier-Stokes equation was used:

$$
\begin{aligned}
\rho_{v} \frac{\partial u_{v}}{\partial t}+\rho_{v}\left(u_{v} \cdot \nabla\right) u_{v}= & \nabla \cdot\left[-p_{v} I+\eta_{v}\left(\nabla u_{v}+\left(\nabla u_{v}\right)^{T}\right)\right. \\
& \left.-\left(\frac{2}{3} \eta-k_{d v}\right)(\nabla \cdot u) I\right] \\
& +\rho_{v} g+F_{\text {st }} \\
\frac{\partial \rho_{v}}{\partial t}+\nabla \cdot\left(\rho_{v} u_{v}\right)= & \dot{m} \delta\left(\frac{1}{\rho_{v}}-\frac{1}{\rho_{l}}\right) .
\end{aligned}
$$

The following heat transfer equation was implemented for both phases:

$$
\rho C_{p} \frac{\partial T}{\partial t}+\rho C(u \cdot \nabla) T=-\nabla \cdot k \nabla T+\frac{\dot{m} \delta h_{l v}}{M},
$$

where $C_{p}$ is the fluid specific heat and $k$ is the fluid thermal conductivity.

2.3. Boundary Condition. The boundary conditions for condensation model are rather complicated due to moving interface between liquid and vapor. It is important to realize that liquid and vapor velocities are not necessarily equal at interface:

$$
u_{\mathrm{int}}=u_{l}-\frac{\dot{m}}{\rho_{l}} n,
$$

where $n$ is the unit normal vector to the interface directed from the liquid phase to the vapor phase and $\dot{m}$ is the rate of condensation.

Implemented natural boundary conditions on the interface for the vapor and liquid phases were

$$
\begin{aligned}
n \cdot \rho_{v} u_{v}=\dot{m}\left(1-\frac{\rho_{v}}{\rho_{l}}\right)+\left(n \cdot \rho_{v} u_{l}\right), \\
n \cdot\left[-p_{l} I+\eta_{l}\left(\nabla u_{l}+\left(\nabla u_{l}\right)^{T}\right)\right] \\
=\dot{m}\left(u_{l}-u_{v}\right)+\gamma k n \\
\quad+n \cdot\left[-p_{v} I+\eta_{v}\left(\nabla u_{v}+\left(\nabla u_{v}\right)^{T}\right)\right] .
\end{aligned}
$$

2.4. Mesh. Free meshes with advanced triangular elements were used to mesh the two-dimensional space in which small droplets are formed on the cooled surface. Elements size near the cold surface was much smaller than that of the bulk elements due to droplet formation. Ratio of elements size increasing was set as 1.2, to avoid possible errors such as permeating droplets into bulk area with coarser mesh size.

\section{Results}

3.1. Validation of Simulation Result. The simulated value of heat flux is the base for calculation of steam condensation heat transfer coefficient:

$$
h=\frac{q}{\Delta T} .
$$




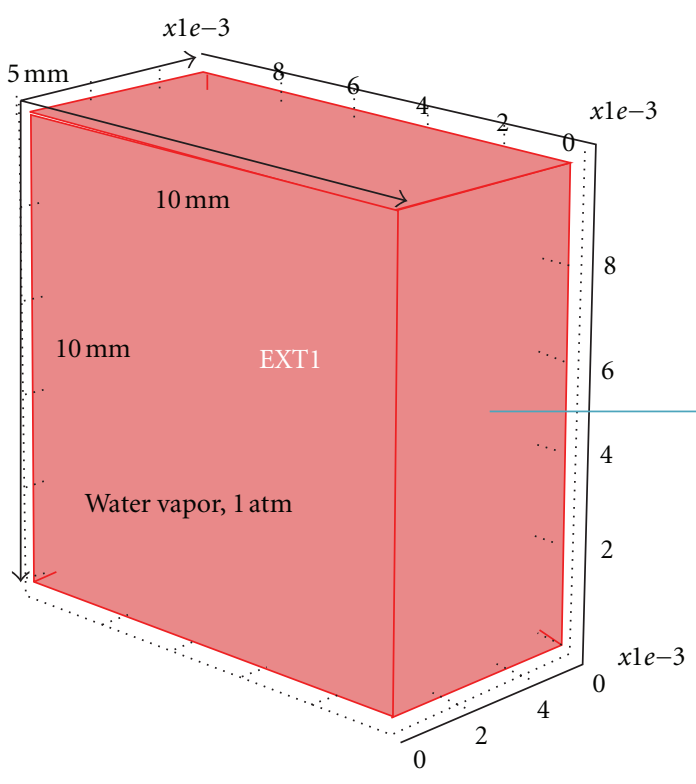

(a)

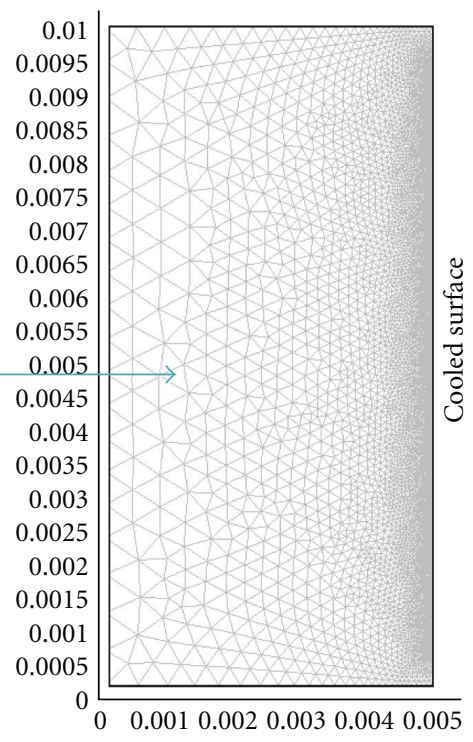

(b)

FIgURE 1: A schematic of the selected system: (a) geometry, (b) grid.

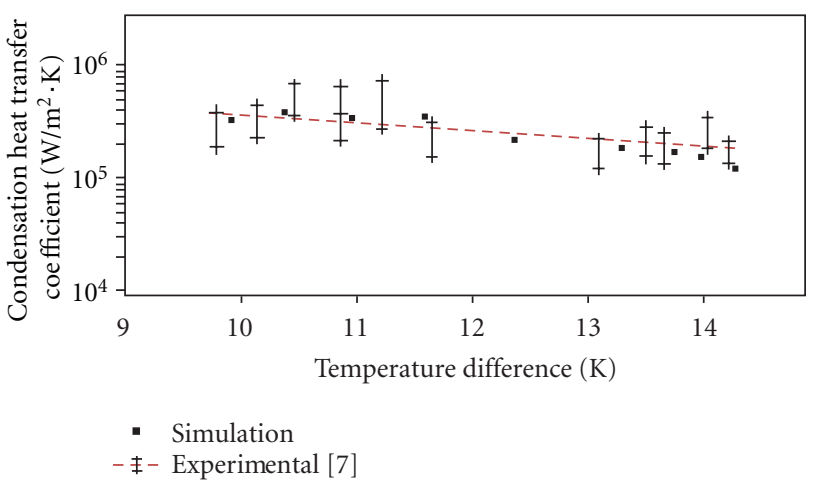

FIGURE 2: Dropwise condensation heat transfer coefficient of steam on PTFE vertical surface at $1 \mathrm{~atm}$ with $\theta=106^{\circ}$, [7].

The simulation results of steam condensation heat transfer coefficient are shown in Figure 2. There is a good agreement between experimental and simulation data. The condensation heat transfer coefficient was decreased by increasing the vapor-surface temperature difference. This is due to the formation coarser size of droplets and lower number of them. Consequently, by increasing the thickness of condensate over the cooled surface, the heat transfer resistance increases.

3.2. Formation of Droplets over Cooled Surface. A large number of droplets with average diameter of 40 micrometer were formed over the cooled surface while the saturated vapor was in the vicinity of the surface. Liquid volume fraction of water droplets on the surface is shown in Figure 3.

By continuation of vapor condensation over the cold surface, the formed droplets were coalesced, which makes droplets bigger. This is due to the movement of droplets as a result of the existing surface tension forces between the liquid-vapor interfaces Figure 4. The mentioned liquid volume fraction in Figures 3 and 4 was defined as the proportion of liquid volume to overall volume, which included both vapor and liquid volumes.

By continuing the condensation process, droplets were dropped due to gravity force and vapor-liquid surface tension. By continuation of dropwise condensation process, heat transfer flux through the cooled surface became steady.

3.3. Effect of Droplets Contact Angle. The effect of contact angle on dropwise condensation heat transfer coefficient was analysed through simulation. As shown in Figure 5, heat transfer coefficient is increased by increasing the contact angle under the same conditions. The Griffith relationship does not determine the condensation coefficient exactly as it does not consider the effect of contact angle. Furthermore, application of this relationship is limited to steam.

One can divide Figure 5 into three parts. In the first part, the temperature difference is less than $5^{\circ} \mathrm{C}$, in which due to low condensation rate and droplet formation, slope of heat transfer coefficient variation is low. Moreover, in this part, condensation coefficient for all contact angles is relatively low, resulting from little temperature difference followed by lack in driving force, and it significantly decreases when temperature difference tends to zero. By increasing temperature difference, condensation coefficient increases due to more formation of droplets on surface, which led to increasing heat transfer surface area. In the second part, where temperature difference is in the range of 5 to $10^{\circ} \mathrm{C}$, condensation is fully dropwise and heat transfer coefficient has its maximum value. In the third part where temperature difference is more than $11^{\circ} \mathrm{C}$, condensation heat transfer coefficient decreases 


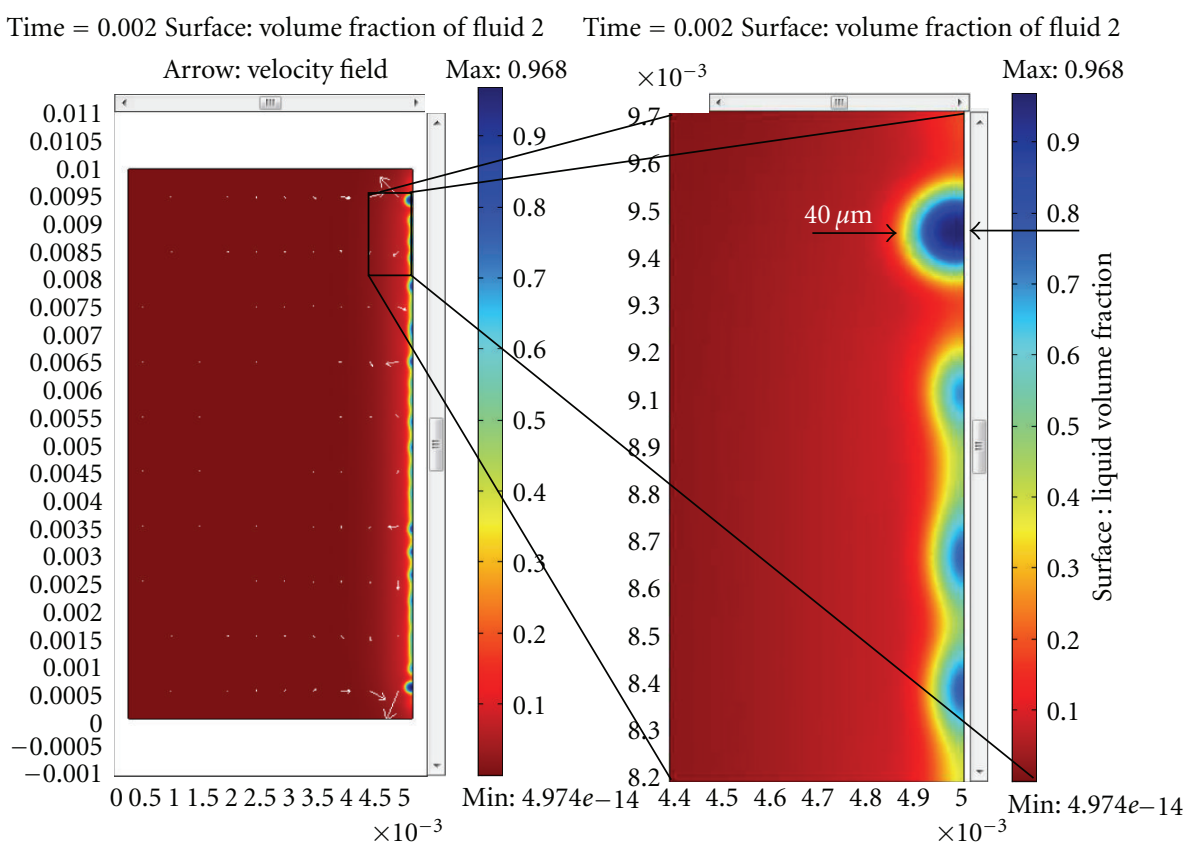

FIgURE 3: A simulated view on formation of droplet over vertical PTFE surface at $1 \mathrm{~atm}$, and enlargement of a section.

due to changing the type of condensation, that is, dropwise to filmwise with higher heat transfer resistance.

\subsection{Dropwise Condensation Heat Transfer Coefficient: A} New Correlation. Not only does dropwise condensation heat transfer coefficient not only depend on the temperature difference between a vapor and cooled surface, but also the contact angle has a crucial role in it. This parameter has not been considered in published relationships. This paper presents a new correlation considering contact angle in order to evaluate condensation heat transfer coefficient more accurately.

In condensation, there are three thermal resistances between vapor and cold surface [3]:

(a) condensate thickness resistance,

(b) vapor-liquid interfacial resistance,

(c) capillary depression (curvature) resistance on the condensate surface.

These three resistances are illustrated in Figure 6.

As shown, the droplet-vapor interface area is $A_{l v}$, and $A_{s l}$ is its contact area with the cold wall. The volume of droplet and the mentioned surface area are [3]

$$
\begin{aligned}
V & =\frac{\pi r^{3}\left(2-3 \cos \theta+\cos ^{3} \theta\right)}{3}, \\
A_{l v} & =2 \pi r^{2}(1-\cos \theta), \\
A_{s l} & =\pi r^{2}\left(1-\cos ^{2} \theta\right),
\end{aligned}
$$

where $\theta$ and $r$ are the droplet contact angle and droplet radius, respectively.
Conductive heat transfer rate in the droplet based on Fourier's law is

$$
\dot{Q}=k_{l} A \frac{\partial T}{\partial r} .
$$

By using (14) and (15), the conductive temperature difference becomes

$$
\Delta T_{\text {conduction }}=\frac{\dot{Q} r}{4 \pi r^{2} k_{l}(1-\cos \theta)} .
$$

The rate of convective heat transfer to the droplet based on Newton's law of cooling is

$$
\dot{Q}=h A \Delta T \text {. }
$$

By substituting equation (13) in equation (17), the interfacial temperature difference is

$$
\Delta T_{\text {interface }}=\frac{\dot{Q}}{2 \pi r^{2} h_{i}(1-\cos \theta)} .
$$

Furthermore the capillary temperature difference is [3]

$$
\Delta T_{\text {curvature }}=\frac{2 \sigma T_{w}}{\rho_{l} h_{l v} r}=\frac{r_{\min }\left(T_{v}-T_{w}\right)}{r} .
$$

Thus, overall temperature difference is summation of the existing temperature differences [2]:

$$
\Delta T=\Delta T_{\text {conduction }}+\Delta T_{\text {interface }}+\Delta T_{\text {curvature }} .
$$

By substituting (16), (18), and (19) into equation (20), the heat transfer rate through single droplet becomes

$$
\dot{Q}=\frac{\pi D^{2}}{2} \Delta T \frac{1-D_{\min } / D}{1 / h_{i}-D / 4 k_{l}}(1-\cos \theta) .
$$




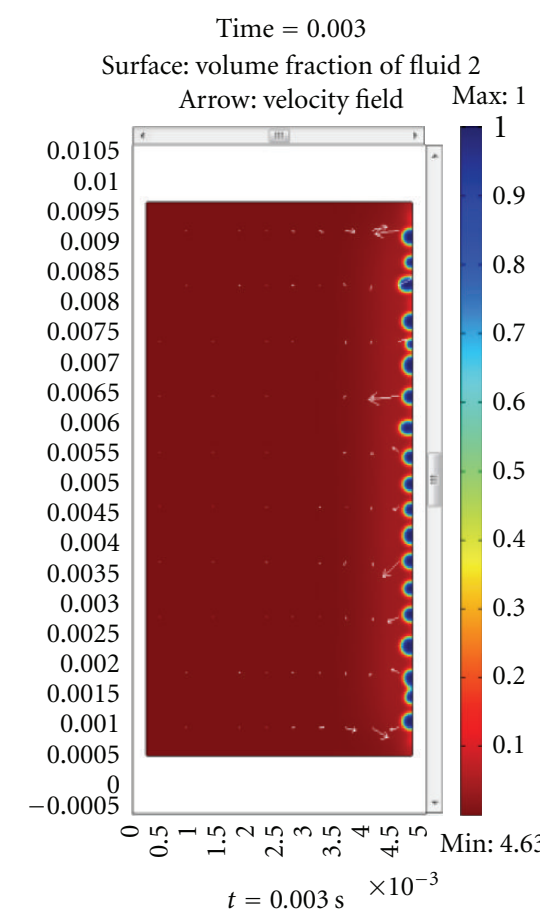

Time $=0.005$

Surface: volume fraction of fluid 2

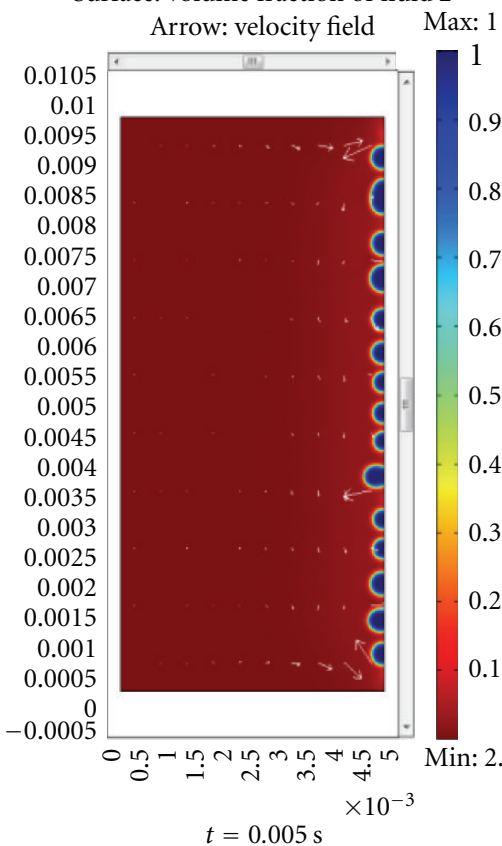

Time $=0.004$

Surface: volume fraction of fluid 2

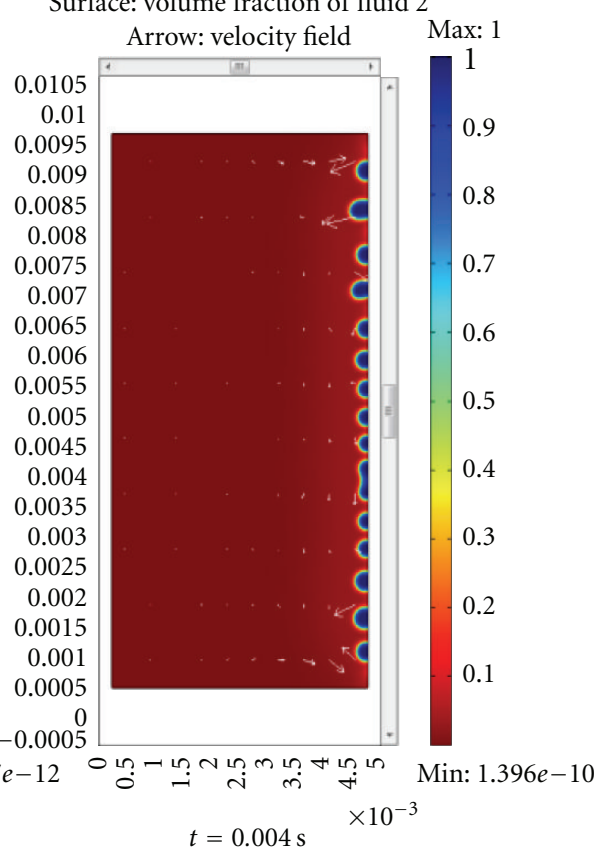

Time $=0.07$

Surface: volume fraction of fluid 2

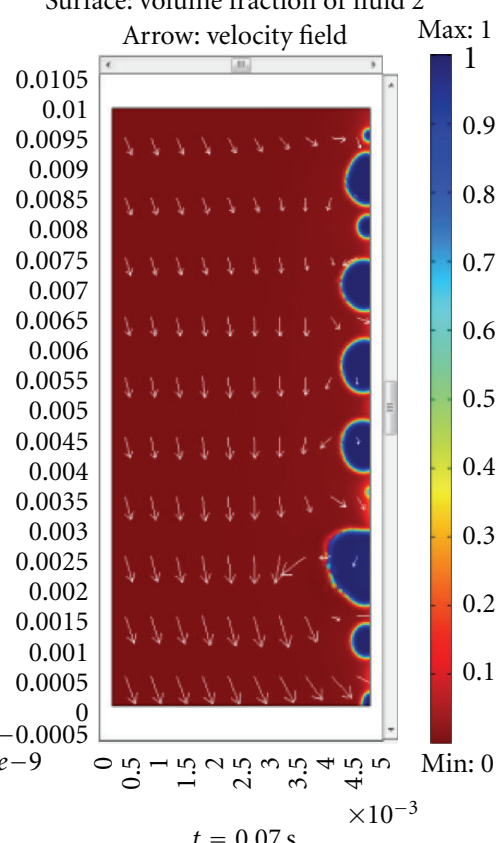

FIGURE 4: Variation of droplets size in time domain.

By integration of (21), which contains droplets size distribution coefficient, $n_{D}^{\prime \prime}$, and with respect to droplets diameter, total heat transfer coefficient of dropwise condensation for unit heat transfer surface can be obtained:

$$
h=\frac{\pi}{2} \int_{D_{\min }}^{D_{\max }} n_{D}^{\prime \prime} D^{2} \frac{\left(1-D_{\min } / D\right)}{\left(1 / h_{i}+D / 4 k_{l}\right)}(1-\cos \theta) d D .
$$

The only difference between relations (22) and (2) is including of the term of $(1-\cos \theta)$. This term shows the rule of contact angle in dropwise condensation heat transfer coefficient, which increases by increasing the contact angle value.

This new relationship can evaluate dropwise condensation heat transfer coefficients more accurately for various 


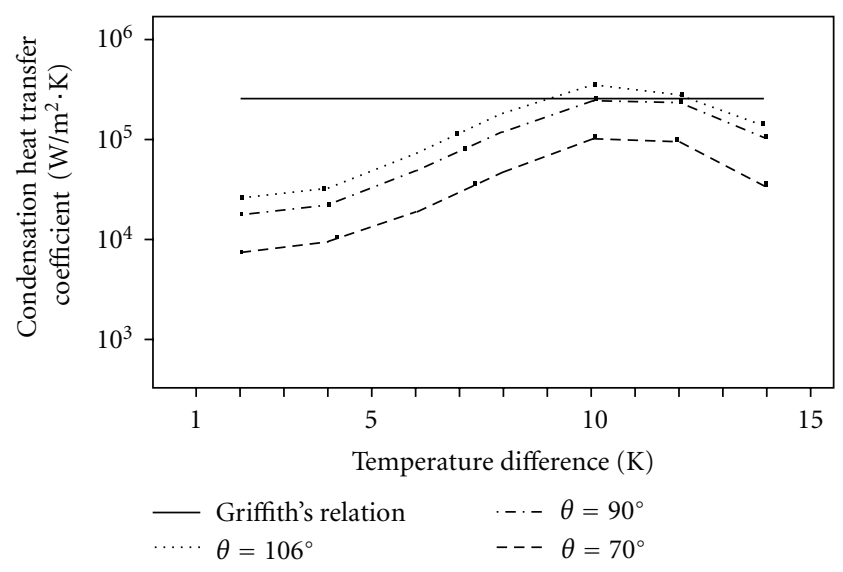

Figure 5: Effect of contact angle on dropwise condensation heat transfer coefficient of steam on various surfaces with different contact angles at $1 \mathrm{~atm}$.

(a)

(b)

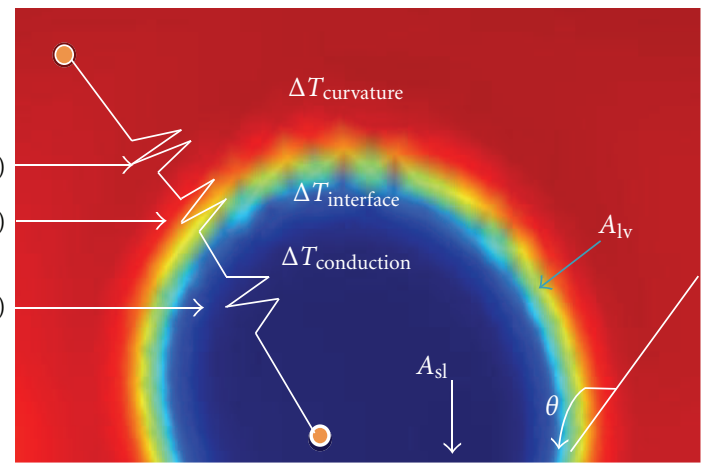

FIGURE 6: A sketch of contact angle and thermal resistances in a droplet.

condensing fluids and surfaces. But, it requires the value of distribution coefficient for formed droplets under operational condition.

3.5. Droplets Size Distribution Coefficient. Droplets size distribution coefficient specifies the number of distributed droplets over unit area of a surface. It is difficult to obtain this value for droplets with diameters less than 10 micrometers [3]. In order to obtain proper value of droplets size distribution coefficient, the finite integral of equation (22) for various temperature differences and contact angles was calculated. Therefore, the value of steam condensation heat transfer coefficient was obtained via numerical simulation. The simulation was performed for different temperature differences of $2,4,6,8,10,12$, and 14 Kelvin and various contact angles of 70, 90, and 106 degrees. By using this value in (22), the only unknown of this equation $\left(n_{D}^{\prime \prime}\right)$ was evaluated. By-three dimensional space regression Figure 7, the optimum relationship for the distribution coefficient was

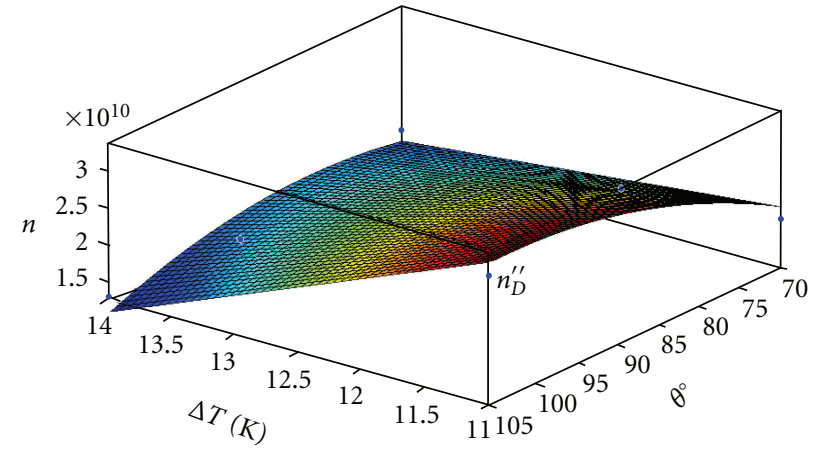

FIgure 7: Regression in 3D space.

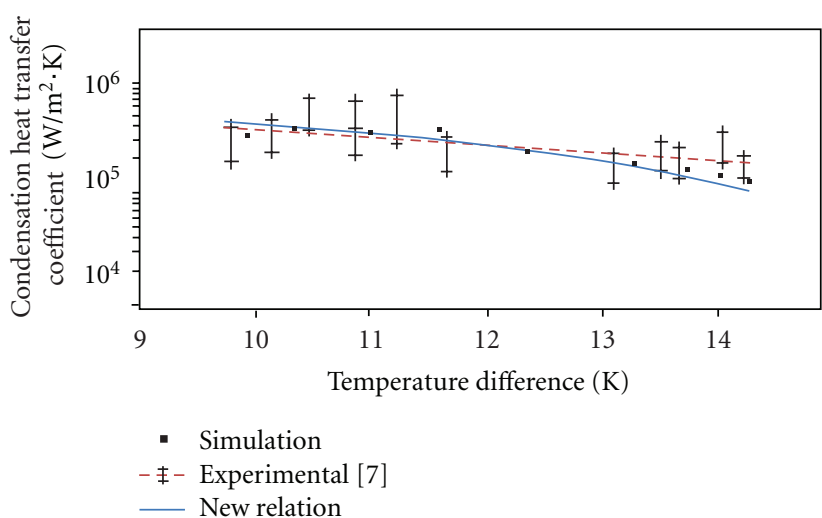

FIGURE 8: Dropwise condensation heat transfer coefficient of steam on PTFE at $1 \mathrm{~atm}, \theta=106^{\circ}$, [7].

determined (droplet/m), (23).

$$
\begin{aligned}
& n_{D}^{\prime \prime}=-1.641 E 11+8.322 E 9 \Delta T \\
&+3.596 E 9 \theta-1.443 E 8 \Delta T \theta-9.644 E 6 \theta^{2} \\
& 11 \mathrm{~K}<\Delta T<14 \mathrm{~K}, 70^{\circ}<\theta<106^{\circ} .
\end{aligned}
$$

As shown in Figure 7, drop size distribution coefficient is increased by increasing the contact angle and decreasing the temperature difference.

By using the value of drop size contribution coefficient, dropwise condensation heat transfer coefficient can be evaluated based on (22). A comparison was made among simulation data, experimental data, and proposed relationship (22) in Figure 8, which indicates good agreement between them, relative error of $11 \%$.

\section{Conclusion}

Surface free energy difference and electric interaction between condensate and cooled surface are the most important parameters in type of condensation. The results of this interaction are represented in contact angle. So the contact angle indicates the role of surface substance in the condensation 
process. Comparison between experimental data and evaluated results from the Griffith equation, which provides steam condensation heat transfer coefficient, without considering contact angle in atmospheric pressure, shows the large deviation among them. This issue specifies the rule of contact angle in calculation of condensation heat transfer coefficient. In order to verify Carey's relation and establish a relation for determination of condensation heat transfer coefficient to consider the contact angle, the droplets size distribution coefficient have to be evaluated by simulation. By evaluating the dropwise condensation heat transfer coefficient and by the optimization of variables, optimum relationship was derived for drop size distribution coefficient. Comparison of the verified relation with experimental data indicates a difference of $11 \%$ that implies existence of a good agreement.

\section{References}

[1] J. P. Hartnett and T. F. Irvine, Advances in Heat Transfer, vol. 21, Academic Press, Philadelphia, Pa, USA, 1991.

[2] G. D. Bansal, S. Khandekar, and K. Muralidhar, "Measurement of heat transfer during drop-wise condensation of water on polyethylene," Nanoscale and Microscale Thermophysical Engineering, vol. 13, no. 3, pp. 184-201, 2009.

[3] A. Faghri, Y. Zhang, and J. Howell, Advanced Heat and Mass Transfer, Global Digital Press, 2010.

[4] V. P. Carey, Liquid-Vapor Phase-Change Phenomena: An Introduction to the Thermophysics of Vaporization and Condensation Processes in Heat Transfer Equipment, Hemisphere, Washington, DC, USA, 1992.

[5] N. K. Battoo, B. S. Sikarwar, S. Khandekar, and K. Muralidhar, "Mathematical modeling and simulation of dropwise condensation and inclined surfaces exposed to vapor flux," in Proceedings of the 20th National and 9th International ISHMTASME Heat and Mass Transfer Conference, Copyright Research Publishing Services, 2010.

[6] P. Griffith, "Dropwise condensation," in Heat Exchanger Design Handbook, E. U. Schlunder, Ed., Hemisphere Publishing, New York, NY, USA, 2 edition, 1983.

[7] M. Xuehu, T. Bai, C. Jiabin, X. Dunqi, and L. Jifang, "Dropwise condensation heat transfer of steam on a polytethfluoroethylene film, Institute of Chemical Engineering, Dalian University of Technology," Journal of Thermal Science, vol. 10, no. 3, 2001. 

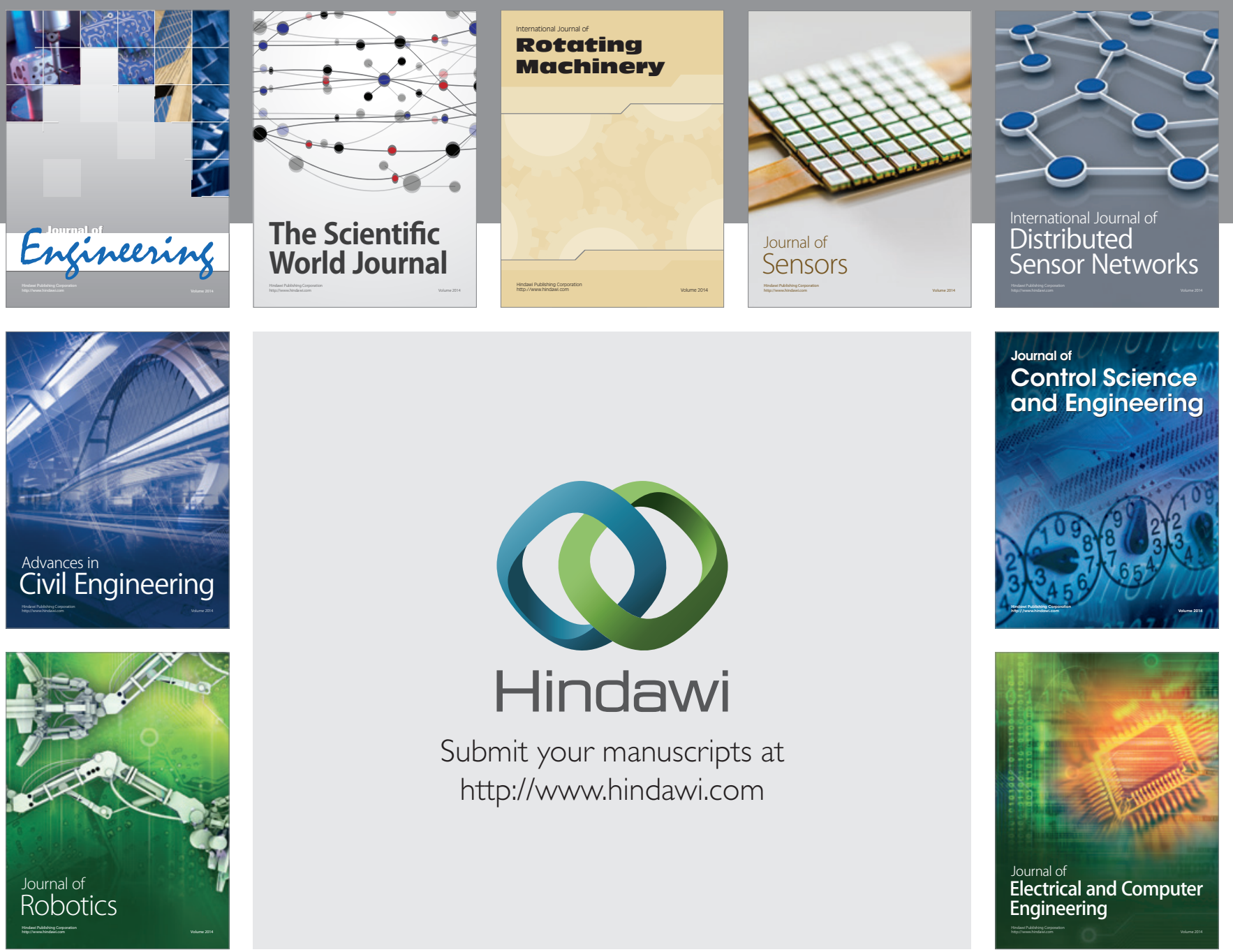

Submit your manuscripts at

http://www.hindawi.com
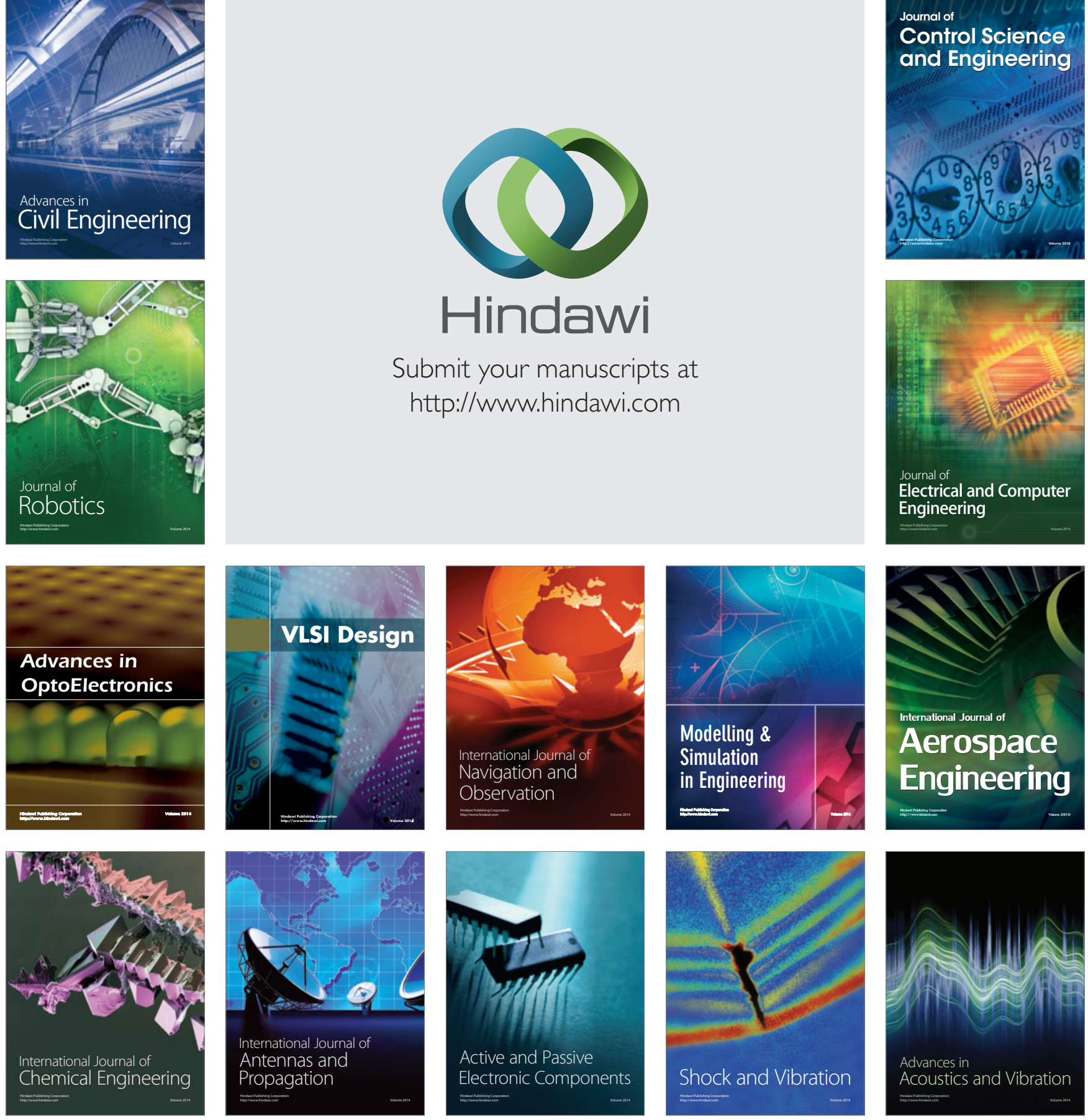